7. H. A. Smith, Tensor products of topological algebras, Dissertation, University of Pennsylvania, Philadelphia, Pa., 1964.

8. J. Tomiyama, Tensor products of commutative Banach algebras, Tôhoku Math. J. 12 (1960), 147-154.

9. T. Turumaru, On the direct-product of operator algebra. I, TOhoku Math. J. 4 (1953), 242-251.

University of Pennsylvania

\title{
SQUARE ROOTS IN BANACH ALGEBRAS
}

\section{TERRELL GARDNER}

A complex number which is not a nonpositive real number has a unique square root in the right half-plane. In this paper, we obtain an extension of this observation to general (complex) Banach algebras.

Since the elements we study are regular, and have logarithms, the existence of square roots is not at stake. Even the existence of roots having the desired spectral properties is evident. The uniqueness result we suppose to be new. It subsumes a somewhat weakened version of the classical result for positive, hermitian, bounded operators on a Hilbert space (that such an operator has a unique positive hermitian and bounded square root): our theorem would apply only to the positive definite (regular) case.

Whether the results of this paper can be extended to the case of not necessarily regular elements of a Banach algebra, we do not at present know.

Definition. A subset $A$ of the complex field will be called positive if $x \in A$ implies $x>0 ; A$ is weakly positive if $x \in A$ implies $\Re(x)>0$. Let $\Sigma$ denote the complement, in the complex plane, of the nonpositive real numbers.

Theorem. Let $\mathfrak{B}$ be a Banach algebra, and $b$ an element of $\mathfrak{B}$ with spectrum $\mathrm{Sp}(b)$ contained in $\Sigma$. Then there exists in $\mathfrak{B}$ a unique square root of $b$ with weakly positive spectrum. If $\mathrm{Sp}(b)$ is real (hence positive), so is $\mathrm{Sp}\left(b^{1 / 2}\right)$.

Proof. Existence: We assume first that $\mathfrak{B}$ has an identity ele-

Received by the editors May 24, 1965. 
ment $e$. Let $z \rightarrow z^{1 / 2}$ denote the principal branch of the square root function in $\Sigma$, and $C$ an oriented envelope of $\operatorname{Sp}(b)$ in $\Sigma$. Set

$$
b^{1 / 2}=(2 \pi i)^{-1} \int_{c} z^{1 / 2}(z e-b)^{-1} d z .
$$

Then $b^{1 / 2}$ so defined is a square root of $b$ with the desired spectral properties [2].

UNIQUENESS: Let $r \in \mathfrak{B}$ with $r^{2}=b$, and $\mathrm{Sp}(r)$ weakly positive. Then $r$ commutes with $b$, and so with any element of the closed subalgebra $\mathfrak{B}^{\prime}$ generated by $b$ and $e$. To prove $b^{1 / 2} \in \mathfrak{B}^{\prime}$, we let $\left(p_{n}\right)$ be a sequence of polynomials in $z$ converging uniformly on a neighborhood of $\mathrm{Sp}(b)$ to $z^{1 / 2}$. (Runge's Theorem.) Then $\left\|p_{n}(b)-b^{1 / 2}\right\| \rightarrow 0$, as desired [2].

Now let $\mathfrak{D}$ be a maximal abelian subalgebra of $\mathscr{B}$ containing $r, b^{1 / 2}$, and $e$. Since the natural injection of $\mathfrak{D}$ into $\mathfrak{B}$ is a spectrum-preserving, isometric isomorphism, we can conduct the remainder of the argument with $\mathfrak{B}$ replaced by $\mathfrak{D}$.

If $h$ is a homomorphism of $\mathfrak{D}$ onto the complex numbers, $h\left(r^{2}\right)$ $=h(b)=h(r)^{2}$, and $\Re h(r)>0$, so $h(r)$ is the unique weakly positive square root of $h(b)$, as is also $h\left(b^{1 / 2}\right)$. Thus $h(r)=h\left(b^{1 / 2}\right)$ for every such $h$, and we have

$$
b^{1 / 2}=r-n, \text { with } \operatorname{Sp}(n)=\{0\} .
$$

Squaring both sides of (1), we obtain $n^{2}=2 r n$, whence, by induction,

$$
n^{2^{k}}=(2 r)^{2^{k}-1} n
$$

From (2),

$$
\begin{aligned}
\left\|n^{2^{k+1}}\right\| 1^{1 / 2^{k+1}} & =\left\|(2 r)^{2^{k+1}-1} n\right\|^{1 / 2^{k+1}}=\left\|\frac{1}{2} 2^{2^{k+1}} r^{2^{k+1}} r^{-1} n\right\|^{1 / 2^{k+1}} \\
& =2^{1-1 / 2^{k+1}}\left\|b^{2^{k}} r^{-1} n\right\|^{1 / 2^{k+1}} .
\end{aligned}
$$

Since the spectral radius of $n$ is $0, \lim _{k \rightarrow \infty}\left\|b^{2^{k}} r^{-1} n\right\| \|^{1 / 2^{k+1}}=0$.

On the other hand, the Banach algebra inequality $\|x y\| \leqq\|x\|\|y\|$ implies $\left\|r^{-1} n\right\| \leqq\left\|b^{-2^{k}}\right\|\left\|b^{2^{k}} r^{-1} n\right\|$ and $\left\|b^{-1}\right\| 2^{k} \geqq\left\|\left(b^{-1}\right)^{2^{k}}\right\|$, whence

$$
\left\|b^{-1}\right\|-2^{k}\left\|r^{-1} n\right\| \leqq\left.\left\|\left(b^{-1}\right)^{2^{k}}\right\|\right|^{-1} \cdot\left\|r^{-1} n\right\| \leqq\left\|b^{2^{k}} r^{-1} n\right\|,
$$

so that $\left\|b^{-1}\right\|-1 /\left.2\left\|r^{-1} n\right\|\right|^{1 / 2^{k+1}} \leqq\left\|b^{2^{k}} r^{-1} n\right\|^{1 / 2^{k+1}}$. Since the right side of the last inequality tends to zero, we must have $r^{-1} n=0$, so $n=0$, and $b^{1 / 2}=r$, which was to be shown. 
Finally, if $\mathfrak{B}$ is without identity element, we adjoin one. A trivial computation then shows that for $b \in \mathfrak{B}, b^{1 / 2} \in \mathfrak{B}$, also.

The theorem can be applied to simplify the proofs in [1].

Added in proof. The reader's attention is directed to the elegant study by Hille, On roots and logarithms of elements of a complex Banach algebra, Math. Ann. 136 (1958), 46-57, which came to the author's attention after this work was completed. Our theorem can be obtained easily from Hille's Theorem 1 .

\section{REFERENCES}

1. L. T. Gardner, An invariance theorem for representations of Banach algebras, Proc. Amer. Math. Soc. 16 (1965), 983-986.

2. E. Hille and R. S. Phillips, Functional analysis and semi-groups, Amer. Math. Soc. Colloq. Publ. Vol. 31, Amer. Math. Soc., Providence, R. I., 1957.

Queens College 